\title{
Optimization of DSC MRI Echo Times for CBV Measurements Using Error Analysis in a Pilot Study of High-Grade Gliomas
}

\author{
(D)L.C. Bell, (D) M.D. Does, (D)A.M. Stokes, (D).C. Baxter, (DK.M. Schmainda, (D)A.C. Dueck, and (D)C.C. Quarles
}

\begin{abstract}
BACKGROUND AND PURPOSE: The optimal TE must be calculated to minimize the variance in CBV measurements made with DSC MR imaging. Simulations can be used to determine the influence of the TE on CBV, but they may not adequately recapitulate the in vivo heterogeneity of precontrast T2*, contrast agent kinetics, and the biophysical basis of contrast agent-induced T2* changes. The purpose of this study was to combine quantitative multiecho DSC MRI T2* time curves with error analysis in order to compute the optimal TE for a traditional single-echo acquisition.
\end{abstract}

MATERIALS AND METHODS: Eleven subjects with high-grade gliomas were scanned at $3 T$ with a dual-echo DSC MR imaging sequence to quantify contrast agent-induced $\mathrm{T}^{*}$ changes in this retrospective study. Optimized TEs were calculated with propagation of error analysis for high-grade glial tumors, normal-appearing white matter, and arterial input function estimation.

RESULTS: The optimal TE is a weighted average of the T2* values that occur as a contrast agent bolus transverses a voxel. The mean optimal TEs were $30.0 \pm 7.4 \mathrm{~ms}$ for high-grade glial tumors, $36.3 \pm 4.6 \mathrm{~ms}$ for normal-appearing white matter, and $11.8 \pm 1.4 \mathrm{~ms}$ for arterial input function estimation (repeated-measures ANOVA, $P<.001$ ).

CONCLUSIONS: Greater heterogeneity was observed in the optimal TE values for high-grade gliomas, and mean values of all 3 ROIs were statistically significant. The optimal TE for the arterial input function estimation is much shorter; this finding implies that quantitative DSC MR imaging acquisitions would benefit from multiecho acquisitions. In the case of a single-echo acquisition, the optimal TE prescribed should be 30-35 ms (without a preload) and 20-30 ms (with a standard full-dose preload).

ABBREVIATIONS: AIF = arterial input function; $\mathrm{CA}=$ contrast agent; $\mathrm{NAWM}=$ normal-appearing white matter

D

ynamic susceptibility contrast MR imaging is increasingly used to map cerebral blood volume in patients with brain tumor, owing to its potential to predict treatment response, improve image-guided biopsies, and differentiate posttreatment radiation effects and glioma progression. ${ }^{1-9}$

CBV is typically acquired by using a dynamic susceptibility contrast MR imaging and tracking the gadolinium-based contrast

Received December 2, 2016; accepted after revision May 7, 2017.

From the Division of Imaging Research (L.C. Bell, A.M.S., L.C. Baxter, C.C.Q.), Barrow Neurological Institute, Phoenix, Arizona; Department of Biomedical Engineering (M.D.D.), Vanderbilt University Institute of Imaging Science, Nashville, Tennessee; Departments of Biophysics and Radiology (K.M.S.), Medical College of Wisconsin, Milwaukee, Wisconsin; and Division of Health Sciences Research (A.C.D.), Section of Biostatistics, Mayo Clinic, Scottsdale, Arizona.

This work was supported by the National Institutes of Health/National Cancer Institute R01 CA158079.

Please address correspondence to C. Chad Quarles, PhD, Barrow Neurological Institute, 350 W Thomas Rd, Phoenix, Arizona 85018; e-mail: chad.quarles@barrowneuro.org

- Indicates open access to non-subscribers at www.ajnr.org

7 Indicates article with supplemental on-line photo.

http://dx.doi.org/10.3174/ajnr.A5295 agent (CA)-induced $\mathrm{T} 2{ }^{\star}$ changes with time. To optimally capture the MR signal changes due to the $\mathrm{T} 2^{\star}$ changes, one must prescribe an optimal TE during the acquisition. Previous studies have used simulations to determine optimal TEs for spin-echo-based DSC MR imaging ${ }^{10}$; however, to the best of our knowledge, an optimal TE has not been determined for gradient-echo-based DSC MR imaging. Recently, the American Society of Functional Neuroradiology recommended gradient-echo-based DSC MR imaging for brain tumor imaging because of the higher signal to noise, better sensitivity, and more uniform vessel-size sensitivity compared with spin-echo imaging. ${ }^{11}$ Accordingly, it is important to determine an optimal TE for gradient-echo-based DSC MR imaging.

Optimal TEs have been previously suggested in the literature. It has been suggested that the optimal TE should be on the order of the expected $\mathrm{T}^{*}$ values before CA administration. ${ }^{12,13}$ In contrast, Boxerman et $\mathrm{al}^{10}$ demonstrated, with propagation of error analysis, that the optimal TE should be a weighted average of the precontrast and postcontrast $\mathrm{T} 2{ }^{*}$ values, though they did not rec- 


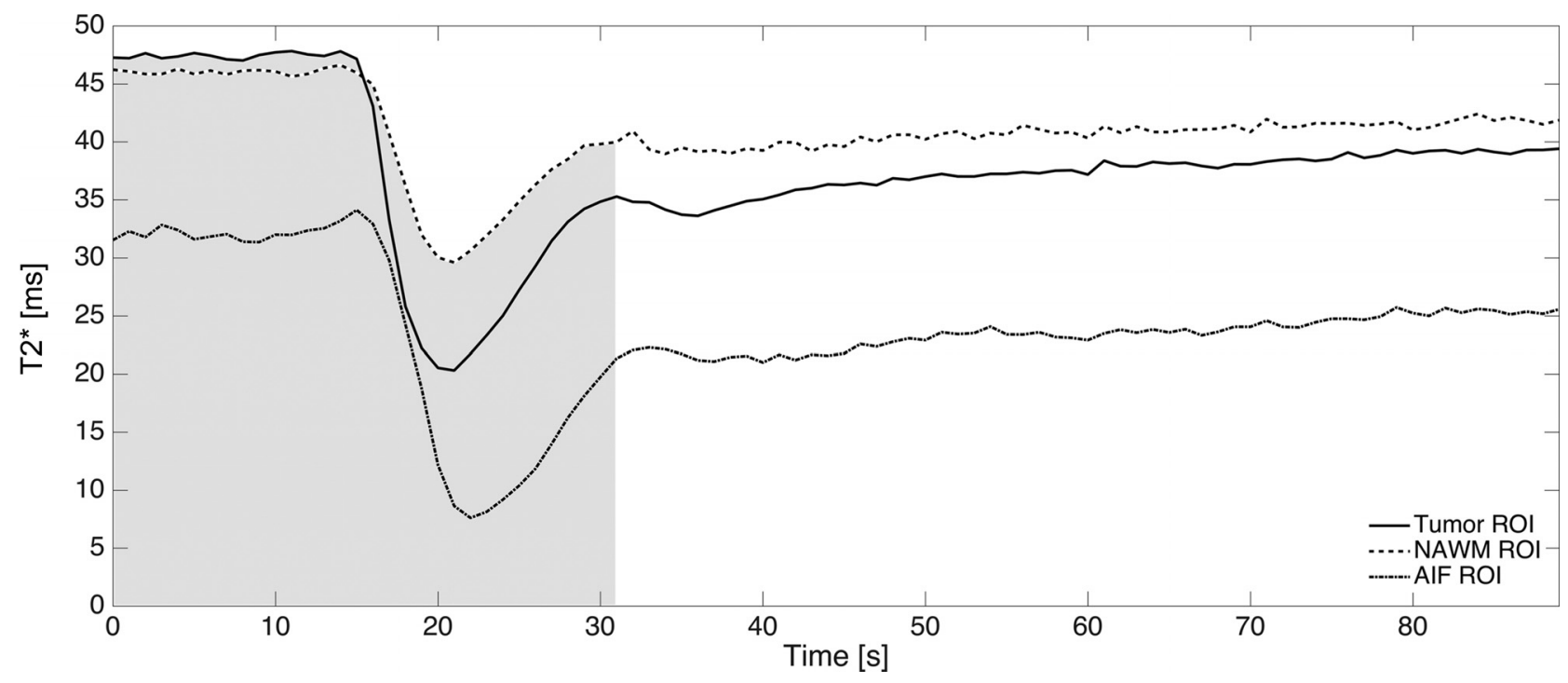

FIG 1. An example from 1 patient of the absolute T2* time curves for tumor (solid), NAWM (dash dash), and the AIF (dash dot) ROIs. As described in Equation 6, the optimal TE is proportional to the weighted average of absolute T2* values during the first pass (as indicated by the shaded gray area) of the contrast agent.

ommend a specific TE. The optimal TE in a DSC experiment is most likely different for voxels used to identify the arterial input function (AIF) and normal-appearing white matter (NAWM) due to the higher contrast agent concentration encountered within arteries and the associated potential signal saturation. ${ }^{14}$

While simulations and error analysis can be used to systematically investigate the influence of TE on CBV accuracy, they cannot recapitulate in vivo heterogeneity of precontrast $\mathrm{T} 22^{*}, \mathrm{CA}$ kinetics, and the biophysical basis of CA-induced $\mathrm{T}^{*}$ changes. With multiecho-based DSC MR imaging acquisitions, voxelwise $\mathrm{T} 2{ }^{*}$ values before and after CA administration can be quantified across tissue types by assuming a monoexponential decay. The goal of this study was to combine quantitative multiecho DSC MR imaging $\mathrm{T}^{*}$ time curves with propagation of error analysis to compute the optimal gradient-echo TEs for high-grade glial tumors, NAWM, and AIF estimation.

\section{MATERIALS AND METHODS}

\section{Theory}

During the contrast agent bolus passage in a DSC MR image, the $\mathrm{T}^{*}$ of the perfused voxel changes with time. From the literature, it is assumed that this change in the transverse relaxation rate, $\Delta \mathrm{R}_{2}^{*}(t)=1 / \Delta \mathrm{T} 2^{*}(t)$, is linear with CA concentration, and therefore the relative $\mathrm{CBV}$ (rCBV) can be approximated by

$$
\mathrm{rCBV}=\int_{0}^{T} \Delta \mathrm{R}_{2}^{*}(t) d t
$$

assuming a single-echo acquisition, $\Delta \mathrm{R}_{2}{ }^{\star}(t)$ is

$$
\Delta \mathrm{R}_{2}^{*}(t)=\mathrm{R}_{2}^{*}(t)-\mathrm{R}_{20}^{*}=-\frac{1}{\mathrm{TE}} \ln \left(\frac{S(t)}{S_{\mathrm{pre}}}\right) .
$$

Here $\mathrm{R}_{20}{ }^{*}$ is the baseline apparent transverse relaxation rate before contrast arrival. The generalized signal equation, after contrast agent injection, for a spoiled gradient-echo acquisition is
3) $S(t)=S 0 \sin \alpha \exp [-\mathrm{TE}$

$$
\begin{aligned}
\left.\times \mathrm{R}_{2}^{*}(t)\right] \frac{1-\exp \left[-\mathrm{TR} \times \mathrm{R}_{1}(t)\right]}{1-\cos \alpha \exp \left[-\mathrm{TR} \times \mathrm{R}_{1}(t)\right]} \\
=\eta \exp \left[-\mathrm{TE} \times \mathrm{R}_{2}^{*}(t)\right] .
\end{aligned}
$$

For simplification, the constant $\eta$ includes all the terms that are independent of the TE.

If we assume that the baseline (precontrast) signal points are acquired with a sufficient signal-to-noise ratio, we note that the variance in the apparent transverse relaxation is mainly due to the variance in the signal with time. If one applies propagation of error to Equation 2, the variance in $\mathrm{R}_{2}{ }^{*}(t)$ is

4)

$$
\sigma_{\mathrm{R}}^{2}=\frac{\mathrm{k} \sigma_{S}^{2} \exp \left[2 \mathrm{TE} \times \mathrm{R}_{2}^{*}(t)\right]}{\mathrm{TE}^{2}},
$$

where $\mathrm{k}$ is a proportionality constant. Similar to the approach taken by Boxerman et $\mathrm{al},{ }^{10}$ the variance in a CBV measurement can then be determined by

5) $\sigma_{\mathrm{CBV}}^{2}=\Delta t \sum_{i=1}^{N} \sigma_{\mathrm{R}}^{2}\left(t_{\mathrm{i}}\right)=\frac{\mathrm{k} \sigma_{S}^{2} \Delta t}{\mathrm{TE}^{2}} \sum_{i=1}^{N} \exp \left[2 \mathrm{TE} \times \mathrm{R}_{2}^{*}\left(t_{\mathrm{i}}\right)\right]$.

Last, the derivative of Equation 5 is taken with respect to TE and solved when equal to zero to determine the optimal TE that minimizes the variance in CBV measurements:

6)

$$
\mathrm{TE}_{\mathrm{opt}}=\frac{\sum_{i=1}^{\mathrm{N}} \exp \left[2 \mathrm{TE}_{\mathrm{opt}} \times \mathrm{R}_{2}^{*}\left(t_{\mathrm{i}}\right)\right]}{\sum_{i=1}^{\mathrm{N}} \mathrm{R}_{2}^{*}\left(t_{\mathrm{i}}\right) \exp \left[2 \mathrm{TE}_{\mathrm{opt}} \times \mathrm{R}_{2}^{*}\left(t_{\mathrm{i}}\right)\right]} .
$$

The optimal TE can now be solved numerically from Equation 6. Note that the optimal TE is, essentially, the weighted average of $\mathrm{T} 2{ }^{\star}$ values during the contrast agent passage (illustrated by Fig 1).

AJNR Am J Neuroradiol 38:1710-15 Sep 2017 www.ajnr.org 1711 


\section{MR Imaging Protocol}

This article is a retrospective analysis from 2 separate subject cohorts approved by the Barrow Neurological Institute's (1st subject cohort) and Vanderbilt University's (2nd subject cohort) institutional review boards. Both cohorts of patients had World Health Organization grade III and IV primary highgrade gliomas undergoing preoperative imaging for surgical resection. All patients had contrast-enhancing lesions.

We analyzed 2 separate subject cohorts to evaluate optimal TE values in scans acquired with and without a contrast agent preload. In the clinic, a preload ranging from 0.025 to $0.1 \mathrm{mmol} / \mathrm{kg}$ is typically administered 6 minutes before the DSC imaging to reduce CA-induced T1 leakage effects. The first cohort of subjects was used for calculation of optimal TE (described in detail below) without a preload. The second cohort of subjects was scanned with multiecho DSC MR imaging for 7.5 minutes, making it an ideal dataset to quantify $\mathrm{T} 2{ }^{\star}$ changes 6 minutes after contrast agent injection, mimicking a preload situation in the clinic. These results allowed us to understand how a preload would affect the calculated optimal TE.

The first subject cohort consisted of 11 datasets randomly selected from an ongoing study (mean age, $49.9 \pm 12.9$ years; 7 men, 4 women). Each subject was scanned on a single 3T MR imaging system (Signa HDx; GE Healthcare, Milwaukee, Wisconsin) with a dedicated 8-channel phased array brain coil. A 2D single-shot dual-echo spiral DSC sequence was used with the following parameters: TR/TE1/TE2 $=1000 / 5.6 / 28 \mathrm{~ms}$, flip angle $=60^{\circ}$, $\mathrm{FOV}=22 \times 22 \mathrm{~cm}$, section thickness $=5 \mathrm{~mm}$, section spacing $=$ $0 \mathrm{~mm}$, number of sections $=16$, and acquired matrix $=128$ (frequency) $\times 128$ (phase). Gadobenate dimeglumine (MultiHance; Bracco Diagnostics, Princeton, New Jersey) was administered at $0.1 \mathrm{mmol} / \mathrm{kg}$ (a standard dose) by using a power injector for each perfusion scan at a rate of $5 \mathrm{~mL} / \mathrm{s}$. The injection occurred 20 seconds after the start of the DSC scan, and the total length of the scan was 3 minutes. Last, T1-weighted spoiled gradientecho images were acquired $\left(\mathrm{TR} / \mathrm{TE}=6.7 / 2.8 \mathrm{~ms}\right.$, flip angle $=13^{\circ}$, FOV $=32 \times 24 \mathrm{~cm}^{2}$, section thickness $=2 \mathrm{~mm}$, acquired resolution $=0.51 \times 0.51 \times 2 \mathrm{~mm}^{3}$ ) after the perfusion scan to delineate enhancing tumor and NAWM ROIs.

The second subject cohort consisted of 5 datasets (mean age, $47.0 \pm 7.2$ years; 4 men, 1 woman) acquired by using a combined spin- and gradient-echo DSC sequence. Each subject was scanned on a single 3T MR imaging system (Achieva; Philips Healthcare, Best, the Netherlands) with a 32-channel head coil. A 2D singleshot spin- and gradient-echo DSC sequence was used with the following parameters: 2 gradient-echoes, 2 asymmetric spinechoes, and 1 true spin-echo, $\mathrm{TR}=1800 \mathrm{~ms}$, TE1-TE5 $=8.8 / 26$ / $55 / 72 / 90 \mathrm{~ms}$, flip angle $=90^{\circ}, \mathrm{FOV}=24 \times 24 \mathrm{~cm}^{2}$, section thickness $=5 \mathrm{~mm}$, section spacing $=0 \mathrm{~mm}$, number of sections $=15$, and acquired matrix $=76$ (frequency) $\times 76$ (phase). Partial Fourier encoding and sensitivity encoding (acceleration factors 0.73 and 2.0, respectively) were used to obtain acceptable TEs. Gadopentetate dimeglumine (Magnevist; Bayer HealthCare Pharmaceuticals, Wayne, New Jersey) was administered at $0.1 \mathrm{mmol} / \mathrm{kg}$ (a standard dose), with a power injector at a rate of $4 \mathrm{~mL} / \mathrm{s}$. The injection occurred 60 seconds after the start of the DSC scan, and the total length of the scan was 7.5 minutes. Last, 3D T1-weighted spoiled gradient-echo images were acquired $(\mathrm{TR} / \mathrm{TE}=8.9 / 4.6 \mathrm{~ms}$, flip angle $=9^{\circ}, \mathrm{FOV}=25.6 \times 25.6 \times 17.0 \mathrm{~cm}^{3}$, acquired resolution $=1 \times$ $1 \times 1 \mathrm{~mm}^{3}$ ) after the perfusion scan to delineate enhancing tumor and NAWM ROIs.

\section{Postprocessing for Optimal TE Calculation}

The T1-weighted, anatomic data were coregistered to the DSC perfusion data by using rigid registration with the FMRIB Software Library (FSL; http://www.fmrib.ox.ac.uk/fsl). ${ }^{15}$ The DSC perfusion data were analyzed with Matlab (MathWorks, Natick, Massachusetts).

From both the dual-echo spiral and spin- and gradient-echo DSC sequences, $\mathrm{R}_{2}{ }^{*}(t)$ was calculated voxel by voxel by assuming a monoexponential decay in $\mathrm{T} 2{ }^{\star 16}$ :

$$
\mathrm{R}_{2}^{*}(t)=\frac{1}{\mathrm{TE} 2-\mathrm{TE} 1} \ln \left[\frac{S_{\mathrm{TE} 1}(t)}{S_{\mathrm{TE} 2}(t)}\right] .
$$

With this information, the optimal TE was calculated by numerically solving Equation 6 over the first pass of CA. To determine the temporal duration of the first pass, we first calculated the mean whole brain (WB) $\Delta \mathrm{R}_{2}{ }^{*}(t)$ to determine the mean peak time point. The slope of the whole brain $\mathrm{WB} \Delta \mathrm{R}_{2}^{*}(t)$ was then calculated. A positive slope indicated wash-in of $\mathrm{CA}$, whereas a negative slope indicated washout, and a slope of zero, no change in CA. Starting at the peak time point, the time point in which the slope equaled zero was identified and set as the end of the first passage. The first passage of CA was approximately 22.5 seconds from the time of injection across all subjects (see Fig 1 for an illustrative example of the first-pass integration limits).

Three ROIs were used for analysis: tumor, NAWM, and voxels selected for AIF estimation. The tumor and NAWM ROIs were selected by using K-means clustering, with data partitioned into 4 clusters with the T1-weighted anatomic data (On-line Figure). The largest centroid value (brightest signal intensity) consisted of the enhancing tumor along with other unwanted regions such as fat around the skull and eyes. The enhancing tumor was manually selected section by section to separate it from the unwanted regions. The NAWM mask was determined by the second largest centroid value. Finally, voxels with the selected ROIs exhibiting a signal drop of at least 5 SDs from the baseline precontrast signal were used for analysis to ensure sufficient contrast-to-noise for the time curves. The AIF was identified on the DSC perfusion dataset with previously established automated algorithms. ${ }^{17,18}$ This automatic AIF algorithm typically selects AIF pixels in the internal carotid, vertebral, and middle cerebral arteries. All ROI selections were approved by an investigator with $>15$ years of experience in brain tumor imaging (C.C.Q.).

In addition to the above measurements and calculations, we calculated $\mathrm{T}^{\star} 6$ minutes after the CA injection from the second cohort of subjects. The percentage difference in $\mathrm{T} 2^{\star}\left(1 / \mathrm{R}_{2}{ }^{*}\right)$ between precontrast $\mathrm{T}^{\star}(t=0)$ and postcontrast $(t=6$ minutes $)$ was calculated to determine the potential effect of a standard dose $(0.1 \mathrm{mmol} / \mathrm{kg})$ preload on the measured baseline $\mathrm{T} 2{ }^{*}$ values in a DSC scan. The estimated optimal TE with a preload was then calculated by scaling the calculated optimal TE without a preload by the percentage difference in $\mathrm{T} 2{ }^{*}$. 


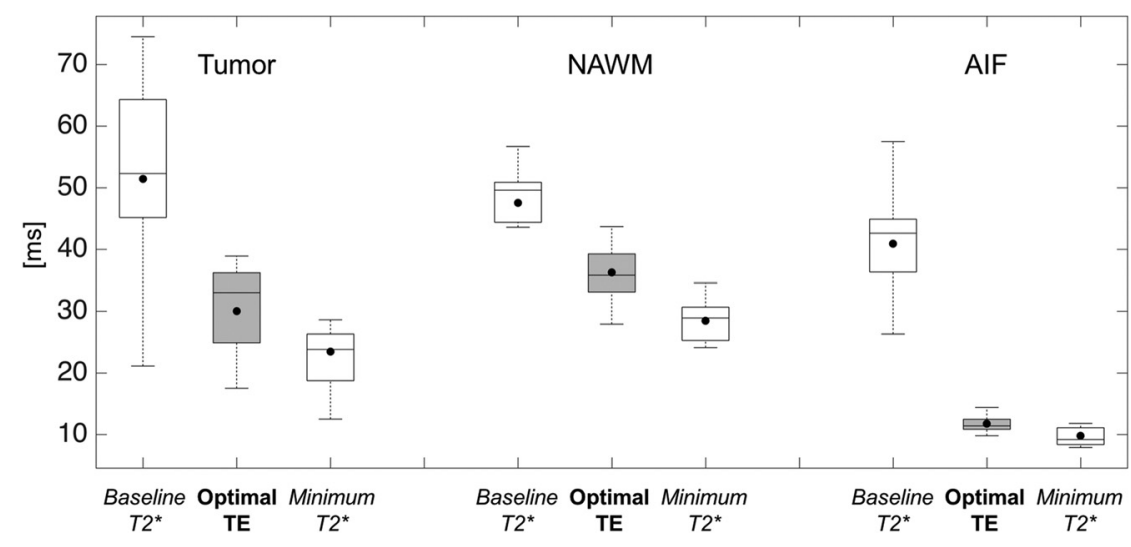

FIG 2. Boxplot representation of baseline and minimum $\mathrm{T}^{*}$ values (white boxplots) along with their respective calculated optimal TE (gray boxplots) in the brain tumor, NAWM, and AIF. Baseline $\mathrm{T}^{*}$ is defined as the $\mathrm{T2}^{*}$ of the ROI before contrast arrival, and the minimum $\mathrm{T}^{*}$ is defined as the $\mathrm{T}^{*}$ value at the peak signal drop. Solid lines represent median values, whereas solid dots represent means across subjects in these boxplots.

\section{Statistical Analysis}

Mean optimal TE values were compared across tumor, NAWM, and AIF ROIs using a multivariate approach with a repeatedmeasures analysis of variance with post hoc pair-wise comparisons using paired $t$ tests. With 11 subjects, this study had an $80 \%$ power to detect a $0.94 \mathrm{SD}$ difference between a pair of ROIs with a 2 -sided $\alpha=.05$ paired $t$ test. To test for mean differences in the minimum $\mathrm{T} 2{ }^{\star}$ of the 2 cohorts of subjects imaged, we performed a Student unpaired $t$ test. Statistical significance was detected for a $P$ value $<.05$.

\section{RESULTS}

Figure 1 illustrates a typical $\mathrm{T}^{*}$ time curve for a single patient in the tumor, NAWM, and AIF. Figure 2 summarizes the baseline and minimum $\mathrm{T}^{*}$ along with the corresponding optimal TE across all 11 subjects from the first cohort. Mean optimal TE values (mean $\pm 1 \mathrm{SD}$ ) were $30.0 \pm 7.4 \mathrm{~ms}$ for tumor, $36.3 \pm 4.6 \mathrm{~ms}$ for NAWM, and $11.8 \pm 1.4 \mathrm{~ms}$ for AIF (repeated-measures ANOVA, $P<.001$; post hoc paired $t$ tests: tumor versus NAWM, $P=.005$; tumor versus AIF, $P<.001$; NAWM versus AIF, $P<$ $.001)$. Tumor ROIs exhibited a wide range of optimal TE values compared with healthy tissue. In all ROIs, mean optimal TE values were closer to the minimum $\mathrm{T}^{*}$ than the baseline values.

Results from the second cohort of subjects indicated that the mean tumor $\mathrm{T}^{\star}$ was $35 \%$ lower than baseline 6 minutes after the CA injection. Additionally, the mean NAWM and AIF T2* were $15 \%$ and $10 \%$, respectively, lower than baseline. If one assumed a respective percentage decrease of each region in $\mathrm{T} 2 * 6$ minutes after CA injection and the optimal TE results from the first cohort of subjects presented above, the estimated mean optimal TE values with a standard preload dose were $19.5 \pm 4.8 \mathrm{~ms}$ for tumor, $30.8 \pm 3.9 \mathrm{~ms}$ for NAWM, and $10.6 \pm 1.3 \mathrm{~ms}$ for AIF.

The concentration of gadolinium between the populations must be similar to apply the results of the second to the first cohort of subjects. Because the concentration of gadolinium is proportional to $\Delta \mathrm{R}_{2}{ }^{*}$, we estimated the similarity between cohorts by comparing the peak $\Delta \mathrm{R}_{2}{ }^{*}$ change. The mean peak $\Delta \mathrm{R}_{2}{ }^{*}$ for the first-versus-second cohort of subjects was $22.9 \pm 12.9$ versus $23.7 \pm 10.8 \mathrm{~ms}$ for tumor $(P=.90), 14.3 \pm 4.2$ versus $12.9 \pm 5.8$ ms for the NAWM $(P=.65)$, and $94.5 \pm$ 18.3 versus $103.2 \pm 30.6 \mathrm{~ms}$ for the AIF $(P=.40)$.

\section{DISCUSSION}

This study determined the optimal TE for a typical single-echo DSC MR imaging acquisition by minimizing the variance in CBV. Using error analysis, we determined that the optimal TE is a weighted average of $\mathrm{T} 2{ }^{*}$ values that occur before and after the CA passage and is dependent on the ROI type.

For an imaging protocol using no preload dose and a standard injection dose at 3T, the optimal TE in brain tumor is $30.0 \pm 7.4 \mathrm{~ms}$. The larger range of optimal TEs in the tumor regions is expected, given the greater variability of blood volume values and CA-induced $\mathrm{T}^{*}$ leakage effects both within and across subjects. ${ }^{19}$ The optimal TE for NAWM is roughly $20 \%$ longer (optimal $\mathrm{TE}=36.3 \pm 4.6 \mathrm{~ms}$ ) than that found in tumor. The optimal TE for the AIF is roughly $300 \%$ shorter (optimal TE $=11.8 \pm 1.4 \mathrm{~ms}$ ) than that found in tumor. For an imaging protocol using both a standard preload and injection dose at 3T, we estimated the optimal TE in brain tumor to be $19.5 \pm 4.8 \mathrm{~ms}$. The estimated optimal TE for NAWM is roughly $65 \%$ longer (optimal $\mathrm{TE}=30.8 \pm 3.9 \mathrm{~ms}$ ) than that found in tumor. The optimal TE for the AIF is roughly $200 \%$ shorter (optimal TE $=10.6 \pm 1.3 \mathrm{~ms}$ ) than that found in tumor. Because a statistically significant difference was detected among the optimal TEs for each of the 3 regions for both imaging options (no preload and preload), multiecho acquisitions are warranted for quantitative DSC MR imaging studies requiring the use of the AIF, supporting previous hypotheses in the literature. ${ }^{20}$

To quantify $\mathrm{T}^{*}$ values throughout the passage of CA in the selected ROIs, we analyzed data from dual-echo spiral acquisitions. The dual-echo data are advantageous because the influence of CA-induced T1 changes that may be present in blood or in situations in which the blood-brain barrier has been compromised has been removed. ${ }^{16}$ Additionally, the calculation of T2* based on dual-echo data is computationally simple. While more TEs could potentially improve the $\mathrm{T} 2^{\star}$ quantification, Stokes and Quarles $^{21}$ have shown that $\mathrm{T} 2^{\star}$ measurements derived from 2 echoes are consistent with those derived from a 5-echo acquisition. Our reported quantitative T2* values for NAWM (47.5 \pm 6.8 $\mathrm{ms}$ ) are within the ranges previously reported in the literature: $48.4 \mathrm{~ms},{ }^{21} 49 \mathrm{~ms},{ }^{22} 50 \pm 8 \mathrm{~ms},{ }^{23}$ and $67.6 \pm 11.0 \mathrm{~ms}^{20}{ }^{20}$ However, comparison of the quantitative $\mathrm{T} 2^{*}$ values for the AIF in this study with those in literature (eg, bulk arterial blood $\mathrm{T} 2{ }^{*}$ ) is difficult because the voxels used to estimate the AIF likely contain brain tissue and arteries due to partial volume effects. Nevertheless, our results indicate that a much shorter optimal TE is needed for AIF estimation, which is consistent with previously published observations. $^{20}$

The optimal TEs reported in this study are specific to a field strength of $3 \mathrm{~T}$ and the CA dose protocol (no preload and 1 stan- 
dard dose of CA). At a different field strength, such as $1.5 \mathrm{~T}$, the optimal TE would be expected to lengthen. A preload of CA is typically administered before the DSC MR imaging to decrease the CA-induced T1 leakage effects. Such preloads could potentially decrease baseline $\mathrm{T}^{*}$ values due to residual CA in the blood and tumor tissue, thereby shortening the optimal TE. To estimate the impact of a standard full dose of preload, we retrospectively analyzed, in a separate cohort of 5 patients with gliomas, multiecho DSC MR imaging data that were acquired for a total of 7.5 minutes. We found that tumor $\mathrm{T}^{*}$ was $35 \%$ lower than baseline 6 minutes after the CA injection (additionally, NAWM was 15\% and AIF was $10 \%$ lower than baseline). If we assume a $35 \%$ decrease in $\mathrm{T}^{*}$ with a preload, the optimal TE will be $35 \%$ shorter than a no-preload-dose scheme or an estimated $19.5 \pm 4.8 \mathrm{~ms}$ for tumor.

There were several limitations to this study. First, our results are specific to $3 \mathrm{~T}$ and to both a standard preload and injection dose. Although $\mathrm{T}^{*}$ is expected to be longer at $1.5 \mathrm{~T}$ than at $3 \mathrm{~T}$, implying that the optimal TE would also increase, we are not able to demonstrate this expectation experimentally. For clinical sites with less than a standard dose for either the preload or injection dose, our results do not apply. However, our derivations and methodology for optimal TE can be easily applied to data acquired at any field strength or dosing scheme of interest. Second, as noted, a statistically significant difference was found among the optimal TEs among tumor, NAWM, and AIF. The extent of using a nonoptimal TE on CBV is unknown. The influence of TE on the accuracy of CBV can be explored both experimentally and through simulations and is currently saved for future work. Third, we have calculated the optimal TE in a small cohort of patients. However, power statistics did demonstrate that our sample size had an $80 \%$ power to detect a 0.94 SD in our measurements. It is unlikely that additional patients would substantially (or practically) shift the calculated optimal TE values. Last, the estimation of the optimal TE from 2 separate cohorts to understand the effect of a preload on the optimal TE is not ideal. Calculation of the optimal TE from multiecho DSC data acquired with a preload would have been ideal. However, clinically, single-echo DSC MR imaging is acquired with a preload, and changes in absolute $\mathrm{T}^{*}$ cannot be obtained from single-echo data for optimal TE calculation. When a multiecho DSC acquisition is acquired in the clinic, a preload is typically not administered because the T1 leakage effects are eliminated. Additionally, our 2 separate cohorts used a standard dose injection of 2 different contrast agents. However, the calculated optimal TE values should not change appreciably because the expected change in $\mathrm{T} 2{ }^{\star}$ due to its relaxation rate is similar ${ }^{22,23}$ across both contrast agents and we found no significant statistical differences in the average gadolinium concentration between the 2 cohorts.

\section{CONCLUSIONS}

The results of this study demonstrate that for conventional singleecho-, gradient-echo-based DSC MR imaging, the optimal TE for CBV mapping in brain tumors and NAWM is $30-36 \mathrm{~ms}$ at $3 \mathrm{~T}$ if no preload has been administered. The accuracy of the AIF will be diminished, to an unknown degree, due to the longer-than-optimal TE. In this situation, CBV should only be calculated in the tumor and the NAWM. If multiple TEs can be prescribed, then a shorter TE of $10 \mathrm{~ms}$ should be prescribed for the AIF and a longer TE of 30-36 ms should be prescribed for the brain tissue and tumor. Given the recommendation of the American Society of Functional Neuroradiology that a preload should be used for DSC MR imaging studies in patients with glioma, the error analysis and patient data described in this study provide experimental evidence that CBV estimates may benefit from lower TEs ( $\sim 20 \mathrm{~ms})$ than the recommended value of $30 \mathrm{~ms}$.

\section{ACKNOWLEDGMENTS}

We acknowledge Kelly Gardener and Samuel McGee for their valuable help.

Disclosures: Laura C. Bell—RELATED: Grant: National Institutes of Health/National Cancer Institute R01 CA158079*. Ashley M. Stokes-RELATED: Grant: National Institute of Health/National Cancer Institute, Comments: R01 CA158079.* Leslie C. Baxter-RELATED: Grant: National Cancer Institute*. Kathleen M. SchmaindaRELATED: Grant: National Institutes of Health, Comments: R01 CA082500, U01 CA176110*; UNRELATED: Other: Imaging Biometrics, Comments: ownership interest. C. Chad Quarles-RELATED: Grant: National Institutes of Health.* Amylou C. Dueck—RELATED: Grant: National Institutes of Health/National Cancer Institute, Comments: CA158079*. *Money paid to the institution.

\section{REFERENCES}

1. Fink JR, Muzi M, Peck M, et al. Multimodality brain tumor imaging: MR imaging, PET, and PET/MR imaging. J Nucl Med 2015;56: 1554-61 CrossRef Medline

2. Boxerman JL, Ellingson BM, Jeyapalan S, et al. Longitudinal DSCMRI for distinguishing tumor recurrence from pseudoprogression in patients with a high-grade glioma. Am J Clin Oncol 2017;40: 228-34 CrossRef Medline

3. Ellingson BM, Zaw T, Cloughesy TF, et al. Comparison between intensity normalization techniques for dynamic susceptibility contrast (DSC)-MRI estimates of cerebral blood volume (CBV) in human gliomas. J Magn Reson Imaging 2012;35:1472-77 CrossRef Medline

4. Chaskis C, Stadnik T, Michotte A, et al. Prognostic value of perfusion-weighted imaging in brain glioma: a prospective study. Acta Neurochir (Wien) 2006;148:277-85; discussion 285 CrossRef Medline

5. Maia AC Jr, Malheiros SM, da Rocha AJ, et al. Stereotactic biopsy guidance in adults with supratentorial nonenhancing gliomas: role of perfusion-weighted magnetic resonance imaging. J Neurosurg 2004;101:970-76 CrossRef Medline

6. Barajas RF Jr, Chang JS, Segal MR, et al. Differentiation of recurrent glioblastoma multiforme from radiation necrosis after external beam radiation therapy with dynamic susceptibility-weighted contrast-enhanced perfusion MR imaging. Radiology 2009;253:486-96 CrossRef Medline

7. Hu LS, Baxter LC, Smith KA, et al. Relative cerebral blood volume values to differentiate high-grade glioma recurrence from posttreatment radiation effect: direct correlation between image-guided tissue histopathology and localized dynamic susceptibility-weighted contrast-enhanced perfusion MR imaging measurements. AJNR Am J Neuroradiol 2009;30:552-58 CrossRef Medline

8. Danchaivijitr N, Waldman AD, Tozer DJ, et al. Low-grade gliomas: do changes in rCBV measurements at longitudinal perfusionweighted MR imaging predict malignant transformation? Radiology 2008;247:170-78 CrossRef Medline

9. Rossi Espagnet MC, Romano A, Mancuso V, et al. Multiparametric evaluation of low grade gliomas at follow-up: comparison between diffusion and perfusion MR with ${ }^{18}$ F-FDOPA PET. Br J Radiol 2016; 89:20160476 CrossRef Medline

10. Boxerman JL, Rosen BR, Weisskoff RM. Signal-to-noise analysis of cerebral blood volume maps from dynamic NMR imaging studies. $J$ Magn Reson Imaging 1997;7:528-37 CrossRef Medline 
11. Welker K, Boxerman J, Kalnin A, et al; American Society of Functional Neuroradiology MR Perfusion Standards and Practice Subcommittee of the ASFNR Clinical Practice Committee. ASFNR recommendations for clinical performance of MR dynamic susceptibility contrast perfusion imaging of the brain. AJNR Am J Neuroradiol 2015;36:E41-51 CrossRef Medline

12. Willats L, Calamante F. The $\mathbf{3 9}$ steps: evading error and deciphering the secrets for accurate dynamic susceptibility contrast MRI. NMR Biomed 2013;26:913-31 CrossRef Medline

13. Thilmann O, Larsson EM, Björkman-Burtscher I, et al. Effects of echo time variation on perfusion assessment using dynamic susceptibility contrast MR imaging at 3 Tesla. Magn Reson Imaging 2004;22:929-35 CrossRef Medline

14. Jochimsen TH, Newbould RD, Skare ST, et al. Identifying systematic errors in quantitative dynamic-susceptibility contrast perfusion imaging by high-resolution multi-echo parallel EPI. NMR Biomed 2007;20:429-38 CrossRef Medline

15. Woolrich MW, Jbabdi S, Patenaude B, et al. Bayesian analysis of neuroimaging data in FSL. Neuroimage 2009;45:S173-86 CrossRef Medline

16. Schmainda KM, Paulson ES, Prah DE, inventors; Imaging Biometrics Llc, assignee. Multiparameter perfusion imaging with leakage correction. US patent 8670602. May 22, 2007

17. Newton AT, Skinner JT, Quarles CC. Automatic AIF estimation in multi-echo DSC-MRI of pediatric patients: avoiding the noise floor. In: Proceedings of the International Society for Magnetic Resonance in Medicine, Salt Lake City, Utah. April 20-26, 2013;21

18. Mouridsen K, Christensen S, Gyldensted L, et al. Automatic selection of arterial input function using cluster analysis. Magn Reson Med 2006;55:524-31 CrossRef Medline

19. Semmineh NB, Xu J, Skinner JT, et al. Assessing tumor cytoarchitecture using multiecho DSC-MRI derived measures of the transverse relaxivity at tracer equilibrium (TRATE). Magn Reson Med 2015;74: 772-84 CrossRef Medline

20. Newbould RD, Skare ST, Jochimsen TH, et al. Perfusion mapping with multiecho multishot parallel imaging EPI. Magn Reson Med 2007;58:70-81 CrossRef Medline

21. Stokes AM, Quarles CC. A simplified spin and gradient echo approach for brain tumor perfusion imaging. Magn Reson Med. 2016; 75:356-62 CrossRef Medline

22. Weinmann HJ, Bauer H, Ebert W, et al. Comparative studies on the efficacy of MRI contrast agents in MRA. Acad Radiol 2002;9:S135-36 CrossRef Medline

23. Pintaske J, Martirosian P, Graf $\mathrm{H}$, et al. Relaxivity of gadopentetate dimeglumine (Magnevist), gadobutrol (Gadovist), and gadobenate dimeglumine (MultiHance) in human blood plasma at $0.2,1.5$, and 3 Tesla. Invest Radiol 2006;41:213-21 CrossRef Medline 